\title{
Dramatic reduction in tumour size in hepatocellular carcinoma patients on thalidomide therapy
}

\author{
Denny Demeria $M D^{1}$, lain Birchall $\mathrm{MBBS}^{2}$, Vincent $\mathrm{G}$ Bain $\mathrm{MD}^{1}$
}

\begin{abstract}
73-year-old man presented with abdominal bloating. His Apast medical history was significant for peptic ulcer disease with partial gastrectomy, hypertension and a remote smoking history. Investigations included contrast-enhanced computed tomography of the abdomen, which revealed innumerable hypervascular lesions, with early enhancement in the arterial phase. These were larger and more frequent in the right lobe of the liver, although they were also present in the left lobe. Most lesions measured $1.0 \mathrm{~cm}$ to $2.0 \mathrm{~cm}$ in diameter but there was a dominant lesion measuring $7.0 \mathrm{~cm} \times 4.1 \mathrm{~cm}$ (Figure 1) in segments 5 and 8 of the right hepatic lobe. No extrahepatic or nodal metastases were identified. Serum alphafetoprotein level was $74.8 \mu \mathrm{g} / \mathrm{L}$ (normal level is less than $9 \mu \mathrm{g} / \mathrm{L}$ ). Core biopsy of the liver confirmed multifocal grade II hepatocellular carcinoma.
\end{abstract}

After having discussed the therapeutic alternatives with the patient, it was concluded that a trial of thalidomide $100 \mathrm{mg}$ once daily, be used. This was obtained through a Health Canada special access program at a cost of $\$ 26.35$ per $50 \mathrm{mg}$ capsule and was continued for one month. Figure 2 shows the post-treatment computed tomography demonstrating that the dominant lesion was no longer hypervascular and was hypoattenuating, consistent with internal necrosis. All the other lesions also appeared necrotic. The patient developed recurrence at the site of the dominant lesion and despite another course of thalidomide, he succumbed to his disease 17 months after diagnosis.

For patients with advanced, multifocal hepatocellular carcinoma, there are no attractive treatment options (1). Tamoxifen has been best studied, but a meta-analysis (2) of seven randomized, controlled trials failed to show either tumour shrinkage or a survival benefit. Octreotide has shown conflicting results in smaller studies and thus, further study is required (3). Similar comments apply to the use of androgens. Chemoembolization has been used as a palliative therapy; however, our patient's large tumour burden precluded this procedure.

There are accumulating data to suggest that antiangiogenic agents may lead to tumour regression in advanced, unresectable hepatocellular carcinoma. The underlying mechanism of action remains to be clearly defined, although data suggests that thalidomide-induced inhibition of basic fibroblast growth factor and vascular endothelial growth factor inhibits endothelial cellular proliferation and microvascular formation $(4,5)$. A small case series (6) in patients with

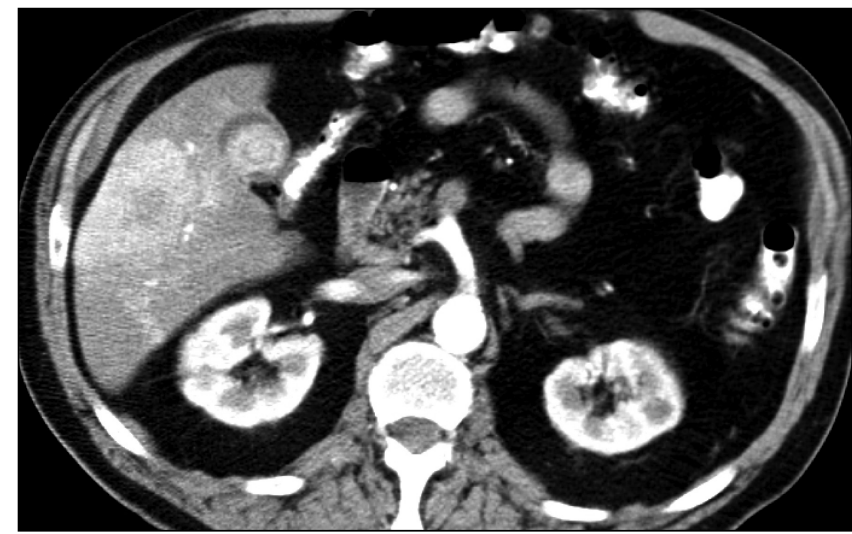

Figure 1) Computed tomography scan of the abdomen revealing multifocal, enhancing lesions including a dominant lesion in the right lobe of the liver

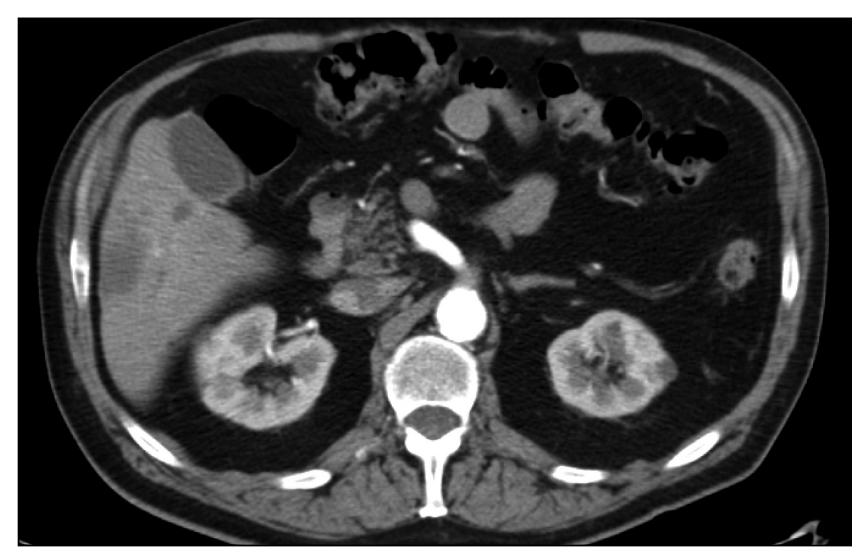

Figure 2) Repeat computed tomography scan of the abdomen after one month of therapy with thalidomide. The dominant tumour and the smaller nodules are now hypoattenuating, consistent with necrosis

advanced hepatocellular carcinoma treated with low-dose thalidomide (200 mg/day to $600 \mathrm{mg} /$ day) had significant reduction in alpha-fetoprotein levels, tumour shrinkage and improved median survival (62.4 weeks, $95 \%$ CI 31.2 to 93.6 weeks in responders versus 18.7 weeks, $95 \%$ CI 11.8 to 25.6 weeks in the entire group that received thalidomide). The results of a phase II clinical trial (7) involving 32 patients found that at doses of $1000 \mathrm{mg} / \mathrm{day}$, there was a $3 \%$ minor

\footnotetext{
${ }^{1}$ Division of Gastroenterology, Department of Medicine; ${ }^{2}$ Department of Diagnostic Imaging, University of Alberta, Edmonton, Alberta

Correspondence: Dr Vincent G Bain, University of Alberta Liver Unit, 1-55 Zeidler Ledcor Center, 130 University Campus, Edmonton, Alberta

T6G 2X8. Telephone 780-492-8128, fax 780-492-8130, e-mail vince.bain@ualberta.ca
}

Received for publication August 28, 2006. Accepted September 5, 2006 
response rate, $3 \%$ partial response rate and a $31 \%$ stable disease rate, while $63 \%$ had disease progression. The overall median survival was 6.8 months. Thalidomide is a relatively simple therapy that the patient can self-administer.

The most common adverse effects include drowsiness, constipation, skin rash and peripheral neuropathy. Although preliminary studies are encouraging, further prospective trials are warranted to evaluate thalidomide in the treatment of advanced hepatocellular carcinoma.

\section{REFERENCES}

1. Schwartz JD, Beutler AS. Therapy for unresectable hepatocellular carcinoma: Review of the randomized clinical trials-II: Systemic and local non-embolization-based therapies in unresectable and advanced hepatocellular carcinoma. Anticancer Drugs 2004;15:439-52.
2. Llovet JM, Bruix J. Systemic review of randomized trials for unresectable hepatocellular carcinoma: Chemoembolization improves survival. Hepatology 2003;37:429-42.

3. Yuen MF, Poon RT, Lai CL, et al. A randomized placebo-controlled study of long-acting octreotide for the treatment of advanced hepatocellular carcinoma. Hepatology 2002;36:687-91. (Erratum in 2003;37:489).

4. Bauer KS, Dixon SC, Figg WD. Inhibition of angiogenesis by thalidomide requires metabolic activation, which is species-dependent. Biochem Pharmacol 1998;55:1827-34.

5. Shiah HS, Chao Y, Chen LT, et al. Phase I and pharmacokinetic study of oral thalidomide in patients with advanced hepatocellular carcinoma. Cancer Chemother Pharmacol 2006;58:654-64.

6. Hsu C, Chen CN, Chen LT, et al. Low-dose thalidomide treatment for advanced hepatocellular carcinoma. Oncology 2003;65:242-9.

7. Patt YZ, Hassan MM, Lozano RD, et al. Thalidomide in the treatment of patients with hepatocellular carcinoma: A phase II trial. Cancer 2005;103:749-55. 


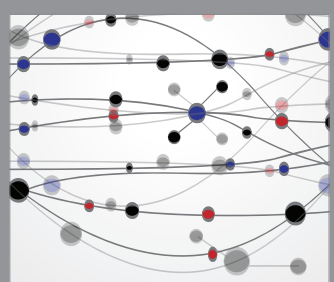

The Scientific World Journal
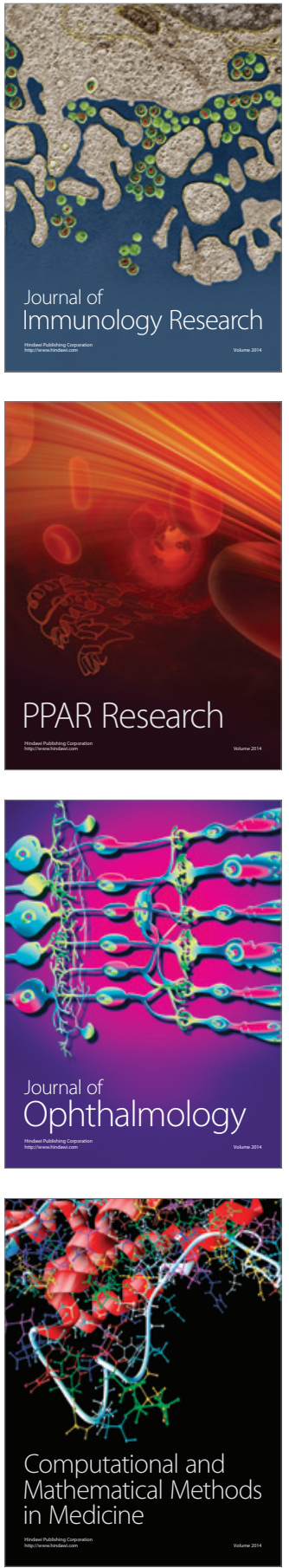

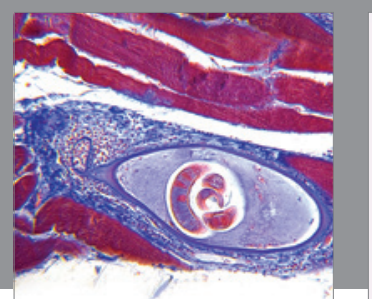

Gastroenterology Research and Practice

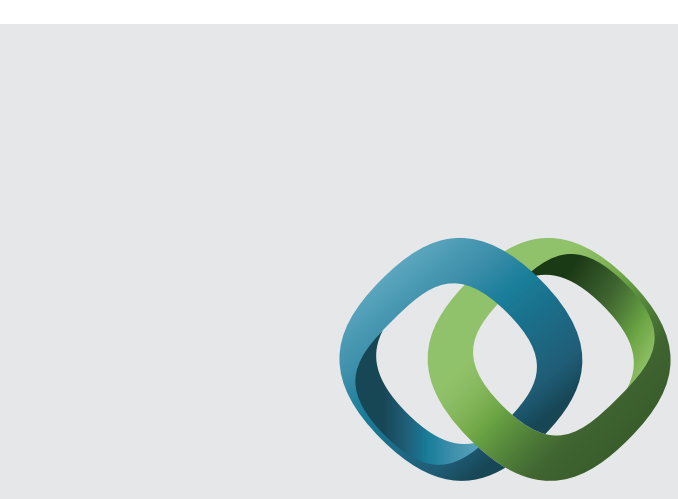

\section{Hindawi}

Submit your manuscripts at

http://www.hindawi.com
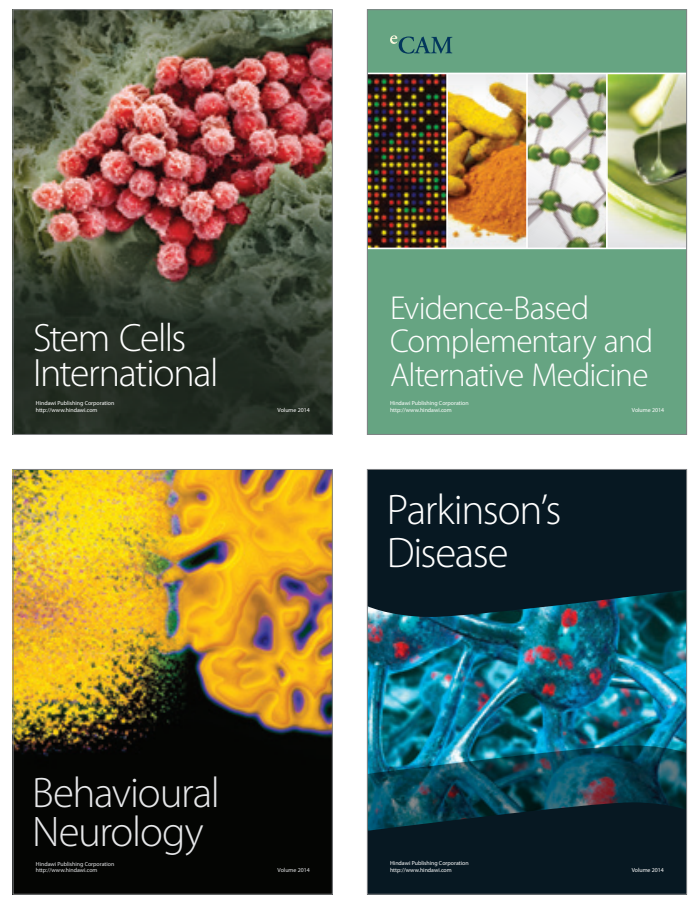
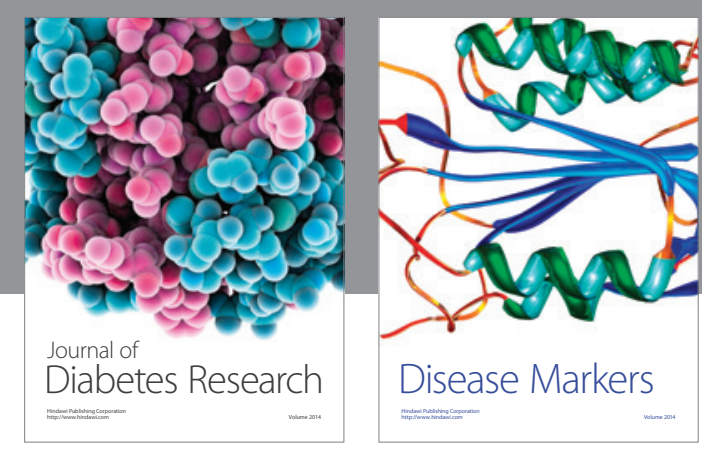

Disease Markers
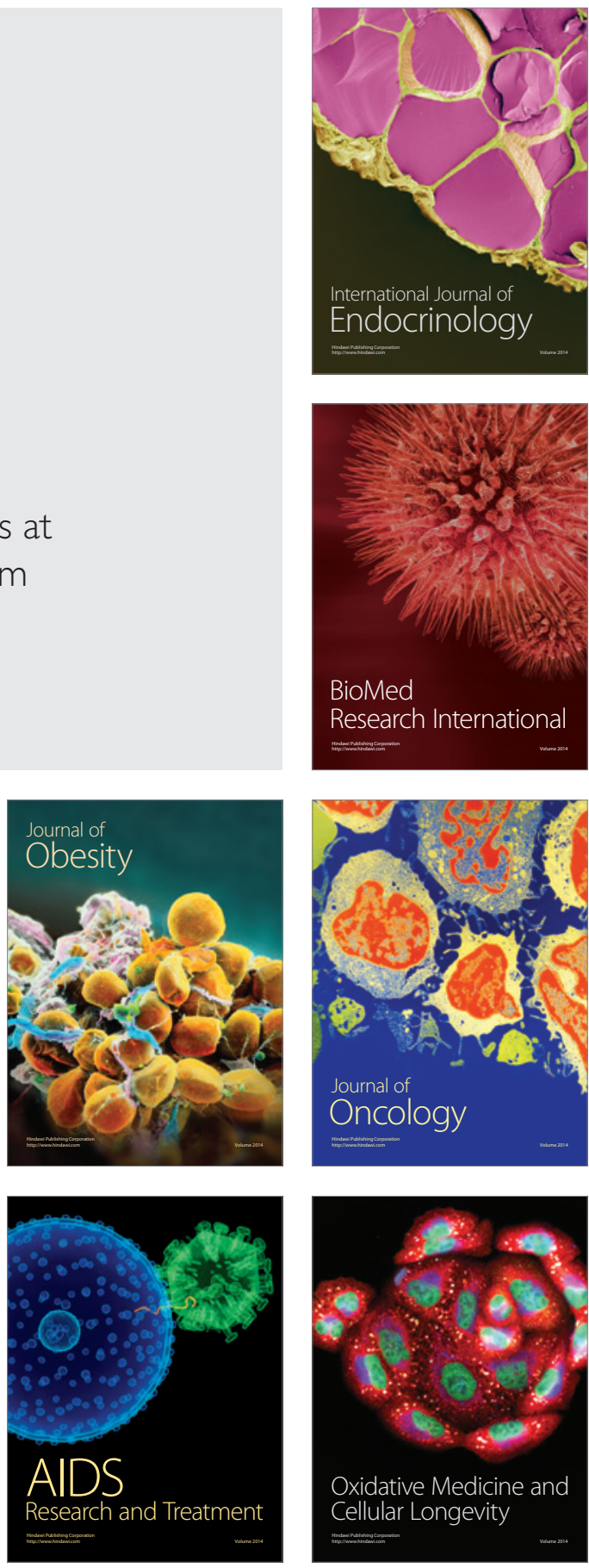